\title{
Genetic background may contribute to the latitude-dependent prevalence of dermatomyositis and anti-TIF1- $\gamma$ autoantibodies in adult patients with myositis
}

\author{
Joanna E. Parkes ${ }^{1 *}$ (D), Simon Rothwell ${ }^{2}$, Alexander Oldroyd $d^{2,3,4}$, Hector Chinoy ${ }^{2,4,5}$, Janine A. Lamb ${ }^{1}$
}

and The Myositis Genetics Consortium (MYOGEN)

\begin{abstract}
Background: The prevalence of dermatomyositis (DM) versus DM and polymyositis (PM) combined has been shown to be negatively associated with latitude. This observation has been attributed to increasing exposure to ultraviolet (UV) light towards the equator. In this study, we investigated whether differing genetic background in populations could contribute to this distribution of DM.

Methods: Case data derived from the MYOGEN (Myositis Genetics Consortium) Immunochip study $(n=1769)$ were used to model the association of DM prevalence and DM-specific autoantibodies with latitude. Control data $(n=9911)$ were used to model the relationship of human leucocyte antigen (HLA) associated with DM autoantibodies and DM or PM single-nucleotide polymorphisms (suggestive significance in the Immunochip project, $P<2.25 \times 10^{-5}$ ) in healthy control subjects with latitude. All variables were analysed against latitude using ordered logistic regression, adjusted for sex.

Results: The prevalence of DM, as a proportion of DM and PM combined, and the presence of anti-transcription intermediary factor 1 (anti-TIF1- $\gamma$ ) autoantibodies were both significantly negatively associated with latitude (OR $0.96,95 \% \mathrm{Cl} 0.95-0.98, P<0.001$; and OR 0.95, 95\% Cl 0.92-0.99, $P=0.004$, respectively). HLA alleles significantly associated with anti-Mi-2 and anti-TIF1- $\gamma$ autoantibodies also were strongly negatively associated with latitude (OR 0.97, 95\% Cl 0.96-0.98, $P<0.001$ and OR $0.98,95 \% \mathrm{Cl} 0.97-0.99, P<0.001$, respectively). The frequency of five PM- or DM-associated SNPs showed a significant association with latitude $(P<0.05)$, and the direction of four of these associations was consistent with the latitude associations of the clinical phenotypes.
\end{abstract}

Conclusions: These results lend some support to the hypothesis that genetic background, in addition to UV exposure, may contribute to the distribution of DM.

Keywords: Dermatomyositis, Polymyositis, Anti-TIF1- $\gamma$, Anti-Mi-2, Latitude, Ultraviolet light

\footnotetext{
* Correspondence: joanna.parkes@postgrad.manchester.ac.uk

${ }^{1}$ Centre for Epidemiology, Division of Population Health, Health Services

Research \& Primary Care, Faculty of Biology, Medicine and Health,

Manchester Academic Health Science Centre, University of Manchester, 2.706

Stopford Building, Oxford Road, Manchester M13 9PT, UK

Full list of author information is available at the end of the article
}

(c) The Author(s). 2018 Open Access This article is distributed under the terms of the Creative Commons Attribution 4.0 International License (http://creativecommons.org/licenses/by/4.0/), which permits unrestricted use, distribution, and reproduction in any medium, provided you give appropriate credit to the original author(s) and the source, provide a link to the Creative Commons license, and indicate if changes were made. The Creative Commons Public Domain Dedication waiver (http://creativecommons.org/publicdomain/zero/1.0/) applies to the data made available in this article, unless otherwise stated. 


\section{Background}

The idiopathic inflammatory myopathies (IIMs) are a spectrum of rare and heterogeneous autoimmune diseases causing inflammation and weakness of skeletal muscle. Major clinical subgroups are polymyositis (PM) and dermatomyositis (DM). The relative prevalence of PM versus DM varies between populations, and it appears to be dependent on latitude; as latitude increases, the relative prevalence of DM (i.e., DM/DM and PM combined) decreases $[1,2]$. This effect has been attributed to higher ultraviolet (UV) radiation levels increasing the likelihood of the development of DM over PM towards the equator, and also increasing the prevalence of the DM-specific autoantibody anti-Mi-2 [2-4]. UV radiation is thought to play a role in DM owing to skin changes being more prominent on the sun-exposed parts of the face, neck and shoulders, reports of photoaggravation of skin lesions and increased cutaneous photosensitivity compared with healthy control individuals $[5,6]$. UV radiation is used in phototherapy of skin conditions such as psoriasis as well as being a major risk factor for skin cancer owing to its suppression of the immune system. UV radiation affects the immune system in various ways, including DNA damage via production of ROS, induction of cytokine signalling and induction of T-regulatory cells [7]. Although thought to be linked to the development of some autoimmune disorders, it is unclear how UV radiation contributes to the pathogenesis of conditions such as DM [2].

Notably, latitude and UV radiation intensity do not perfectly correlate, and UV exposure may depend on the behaviour of individuals, such as whether they work indoors or outdoors. It is possible that the observed differences in DM, PM and anti-Mi-2 prevalence with latitude could additionally be influenced by other factors, such as the different genetic backgrounds of populations. The most strongly associated single-nucleotide polymorphism (SNP) outside the human leucocyte antigen (HLA) gene complex for the PM subgroup in a recent IIM Immunochip study (rs2476601 in PTPN22) [8] demonstrates a correlation with latitude, with increasing risk allele frequency from southern to northern Europe [9]. Similarly, some HLA allele frequencies are known to vary with latitude, such as HLA-B27, associated with ankylosing spondylitis, which is absent in populations around the equator but increases to $40 \%$ in populations in the far north and the Arctic [10].

The aim of this study was to explore the associations of PM and DM prevalence and autoantibody frequencies with latitude. In particular, we investigated whether these "latitude-dependent" associations could instead be attributed to genetic background rather than environmental factors by analysing the distribution of PM and DM risk SNPs in healthy populations.

\section{Methods}

Association of relative prevalence of PM and DM, and DM autoantibody frequency with latitude in a large myositis cohort

Data from the IIM Immunochip study [8] were used to model the association of relative prevalence of PM or DM and DM-specific autoantibody frequency with latitude. Of the DM-specific autoantibodies, only anti-transcription intermediary factor $1-\gamma$ (anti-TIF1- $\gamma$ ) (97 positive, 1345 tested) and anti-Mi-2 (83 positive, 1471 tested) were analysed, because only a limited number of patients were anti-SAE- $(n=29)$ or anti-MDA5-positive $(n=18)$. Autoantibodies were tested in various centres using immunoprecipitation, line blot or enzyme-linked immunosorbent assays. Geographical latitude was assigned according to the approximate latitude of the recruitment centre which contributed samples to the MYOGEN (Myositis Genetics Consortium) Immunochip study as a proxy for latitude of origin and disease onset [8]. Data were available from 1769 cases (914 PM and 855 DM) from 12 countries worldwide (Additional file 1). All individuals included in the study were Caucasian. Juvenile cases were excluded because some contributing centres collected only juvenile DM cases, and this may have introduced bias into the latitudinal associations.

\section{Analysis of association of PM and DM single-nucleotide polymorphism risk alleles with latitude in healthy control subjects}

The risk allele frequencies of ten SNPs associated with PM or DM (suggestive significance threshold of $P<$ $2.25 \times 10^{-5}$ ) in the Immunochip study [8] were analysed for their association with latitude using the control individuals from the study $(n=9911)$. Recruitment centre information was not available for some control individuals; for these samples average latitude of the contributing country was used (see Additional file 1 for details). U.S. control samples lacking recruitment centre information were excluded owing to the large range of possible latitudes.

\section{Analysis of association of HLA alleles with DM-specific autoantibodies with latitude in healthy control subjects} HLA alleles associated with DM-specific autoantibodies also were analysed for their association with latitude in the Immunochip control individuals $(n=9911)$. In the Immunochip data $H L A-D R B 1 * 07: 01$ is strongly associated with anti-Mi-2, and $H L A-D Q B 1 * 02$ is strongly associated with anti-TIF1- $\gamma\left(P=4.36 \times 10^{-12}\right.$ and $P=2.11 \times$ $10^{-11}$ respectively [11], Rothwell S: Personal communication, in preparation). All associations were modelled using ordered logistic regression, adjusted for sex. 
Analysis was carried out using STATA version 13.1 (StataCorp, College Station, TX, USA) [12].

\section{Results}

Relative prevalence of DM and frequency of anti-TIF1- $\gamma$ autoantibodies are significantly negatively associated with latitude in adult myositis

In ordered logistic regression analysis using the IIM Immunochip case data $(n=1769)$, the relative prevalence of DM (versus PM and DM combined) had a significant association with latitude (OR 0.96, 95\% CI 0.950.98, $P<0.001$ ) (Additional file 2). The presence of anti-TIF1- $\gamma$ autoantibodies in the cases tested $(n=1345)$ was negatively associated with latitude (OR 0.95, 95\% CI 0.92-0.99, $P=0.004)$. However, the presence of anti-Mi-2 autoantibodies in the cases tested $(n=1471)$ was not significantly associated with latitude (Table 1 ).

\section{PM and DM SNP risk alleles are significantly associated with latitude in healthy populations}

In ordered logistic regression analysis using data from the Immunochip study controls $(n=9911)$, the frequency of four of eight PM-associated SNP risk alleles showed a significant association with latitude $(P<0.05)$; the three most significant associations were positively associated. One of the two DM-associated SNPs was significantly negatively associated with latitude (Table 2).

\section{HLA alleles associated with DM-specific autoantibodies are significantly associated with latitude in healthy control individuals}

In ordered logistic regression analysis using control data from the IIM Immunochip study $(n=9911)$, HLA alleles associated with the DM-specific autoantibodies anti-Mi-2 and anti-TIF1- $\gamma$ were strongly negatively associated with latitude (OR 0.97, 95\% CI 0.96-0.98, $P<0.001$; and OR 0.98, 95\% CI 0.97-0.99, $P<0.001$, respectively).

\section{Discussion}

In this study we analysed the associations between latitude and DM relative prevalence and DM-specific autoantibody frequencies in the largest IIM cohort to date. In addition, we analysed the association of latitude with PM- or DM-associated SNP risk allele frequencies and HLA alleles associated with DM autoantibodies in healthy populations. The results confirmed that the proportion of DM increases towards the equator, with a strong negative association with latitude. Furthermore, we showed, for the first time, to our knowledge, that DM-specific anti-TIF1- $\gamma$ autoantibody frequency was significantly negatively associated with latitude along with HLA alleles associated with anti-TIF1- $\gamma$ and anti-Mi-2. However, we failed to replicate the increase in the presence of anti-Mi-2 autoantibodies towards the equator which was reported in a previous study $(P=$ 0.0349 ) [2]. The association of anti-Mi-2 with surface UV radiation intensity in that study was more significant $(P=0.0196)$ than the association with latitude and later replicated in another study of UV radiation $[2,3]$. In addition, exposing keratinocytes to UV radiation has been shown to increase the expression of $\mathrm{Mi}-2$ protein [13], supporting the hypothesis that $\mathrm{UV}$ radiation may be associated with production of anti-Mi-2 autoantibodies.

In the analysis of PM- and DM-associated non-HLA SNP risk allele frequency, we found that half of the SNPs analysed were significantly associated with latitude and four of five were consistent with the latitude associations of PM or DM prevalence (positive for PM and negative for DM). Effect sizes were modest, but the ORs had tight CIs and were statistically significant.

This study has some limitations, including the assumption that the patients' location at disease onset and place of birth would be similar to the location of the recruitment centres. The study was limited to one ethnic group because the genetic association study was performed only

Table 1 Association of latitude with dermatomyositis prevalence, dermatomyositis-specific autoantibody and associated human leucocyte antigen allele frequency

\begin{tabular}{|c|c|c|}
\hline \multirow[t]{2}{*}{ Variable } & \multicolumn{2}{|c|}{ Associations with latitude, adjusted for sex } \\
\hline & OR (95\% Cl) & $P$ value \\
\hline Relative prevalence of DM & $0.96(0.95,0.98)$ & $<0.001$ \\
\hline Anti-Mi-2 & $0.99(0.97,1.03)$ & 0.927 \\
\hline Anti-TIF1- $\gamma$ & $0.95(0.92,0.99)$ & 0.004 \\
\hline HLA-DRB1*07:01 (associated with anti-Mi-2) & $0.97(0.96,0.98)$ & $<0.001$ \\
\hline HLA-DQB1*02 (associated with anti-TIF1- $\gamma$ ) & $0.98(0.97,0.99)$ & $<0.001$ \\
\hline
\end{tabular}


Table 2 Association of polymyositis- or dermatomyositis-associated single-nucleotide polymorphisms with latitude

\begin{tabular}{|c|c|c|c|c|c|}
\hline \multirow[t]{2}{*}{ Variable } & \multicolumn{3}{|c|}{ Immunochip associations and $P$ values for subgroups } & \multicolumn{2}{|c|}{ Associations with latitude, adjusted for sex } \\
\hline & Reported gene & Polymyositis & Dermatomyositis & $\mathrm{OR}(95 \% \mathrm{Cl})$ & $P$ value \\
\hline \multicolumn{6}{|c|}{ PM associations } \\
\hline rs2476601 & PTPN22 & $7.90 \times 10^{-11}$ & 0.05 & $1.08(1.05,1.11)$ & $<0.001$ \\
\hline rs4690220 & SLC26A1 | IDUA & $7.47 \times 10^{-6}$ & 0.03 & $1.02(1.01,1.02)$ & 0.001 \\
\hline rs7956536 & $U B E 3 B \mid M M A B$ & $3.66 \times 10^{-6}$ & 0.67 & $1.01(1.00,1.02)$ & 0.004 \\
\hline rs17799348 & FAM167A | BLK & $4.13 \times 10^{-6}$ & 0.32 & $0.99(0.98,0.99)$ & 0.035 \\
\hline rs1420095 & IL18R1 & $6.16 \times 10^{-6}$ & 0.87 & $0.99(0.98,1.01)$ & 0.334 \\
\hline rs2286896 & $N A B 1$ & $3.76 \times 10^{-6}$ & 0.58 & $0.99(0.98,1.01)$ & 0.434 \\
\hline rs9905921 & LOC728073 | RPL38 & $2.01 \times 10^{-6}$ & 0.10 & $0.99(0.99,1.01)$ & 0.748 \\
\hline rs7535818 & RGSI & $1.37 \times 10^{-5}$ & 0.14 & $0.99(0.99,1.01)$ & 0.897 \\
\hline \multicolumn{6}{|c|}{ DM associations } \\
\hline rs4702698 & ROPN1L | ANKRD33B & 0.5 & $4.49 \times 10^{-6}$ & $0.99(0.98,0.99)$ & 0.012 \\
\hline rs570676 & PRR5L | TRAF6 & 0.01 & $6.23 \times 10^{-7}$ & $0.99(0.98,1.00)$ & 0.081 \\
\hline
\end{tabular}

DM Dermatomyositis, $P M$ Polymyositis

All variables were analysed against latitude in an ordered logistic regression analysis, adjusted for sex. Single-nucleotide polymorphisms (SNPs) identified as having suggestively significant $P$ values $\left(P<2.25 \times 10^{-5}\right)$ for either PM or DM in the Immunochip study [8] were used. SNP risk allele frequencies were calculated from the Immunochip study control data $(n=9911)$

in Caucasian samples. In addition, samples were collected within a limited range of latitudes, and the cases and controls were not from exactly the same populations. Studies with collection of disease onset and place of birth data along with UV exposure data in a larger multi-ethnic IIM and control cohort spread over a greater range of latitudes could elucidate whether latitude associations are due to population genetic background or environmental factors.

\section{Conclusions}

Overall, these results lend some support to the hypothesis that different genetic backgrounds of populations may contribute to the increased prevalence of DM toward the equator, in addition to, or instead of, increasing UV radiation exposure levels.

\section{Additional files}

Additional file 1: Table S1. Immunochip study data included in analyses. Assigned latitude and numbers of PM, DM and controls at each centre included in the study. (DOCX $25 \mathrm{~kb}$ )

Additional file 2: Figure S1. The association of relative dermatomyositis (DM) prevalence, frequency of DM-associated autoantibodies, HLA alleles and single-nucleotide polymorphisms with latitude. Two-way linear prediction plot with 95\% Cls was produced using STATA version 13.1 software. Data for DM as a proportion of PM and DM $(n=1769)$, frequency of anti-TIF1- $\gamma$ in all samples tested $(n=1345)$ and frequency of anti-Mi-2 in all samples tested $(n=1471)$ are derived from the Immunochip study [8]. Frequencies of HLA-DQB1*02, HLA-DRB1*07:01, rs4702698 and rs570676 are derived from the Immunochip control data $(n=9911)$. (TIF $2203 \mathrm{~kb})$

\section{Abbreviations}

DM: Dermatomyositis; HLA: Human leucocyte antigen; IIM: Idiopathic inflammatory myopathy; MYOGEN: Myositis Genetics Consortium;
PM: Polymyositis; SNP: Single-nucleotide polymorphism; TIF1- $\gamma$ : Transcription intermediary factor 1-Y; UV: Ultraviolet

\section{Acknowledgements}

The MYOGEN study investigators, in addition to the authors of this article, who contributed to this study are as follows: Ingrid E. Lundberg (Rheumatology Unit, Department of Medicine, Karolinska Institutet, Stockholm, Sweden), Frederick W. Miller (Environmental Autoimmunity Group, Clinical Research Branch, National Institute of Environmental Health Science, National Institutes of Health, Bethesda, MD, USA), Robert G. Cooper (MRC/ARUK Institute of Ageing and Chronic Disease, University of Liverpool, UK), William E. Ollier (Division of Population Health, Health Services Research and Primary Care, School of Health Sciences, Faculty of Biology, Medicine and Health, Manchester Academic Health Science Centre, The University of Manchester, UK), Peter K. Gregersen (The Robert S Boas Center for Genomics and Human Genetics, Feinstein Institute for Medical Research, Manhasset, NY, USA), Jiri Vencovsky (Institute of Rheumatology and Department of Rheumatology, 1st Faculty of Medicine, Charles University, Prague, Czech Republic), Katalin Danko (Division of Clinical Immunology, Department of Internal Medicine, University of Debrecen, Debrecen, Hungary), Vidya Limaye (Rheumatology Unit, Royal Adelaide Hospital and University of Adelaide, Adelaide, Australia), Albert Selva-O'Callaghan (Department of Internal Medicine, Vall d'Hebron General Hospital, Barcelona, Spain), Pedro M. Machado, Michael G. Hanna (MRC Centre for Neuromuscular Diseases, UCL Institute of Neurology, London, UK), Hazel Platt (Centre for Integrated Genomic Medical Research, University of Manchester, UK), Øyvind Molberg (Department of Rheumatology, Oslo University Hospital, Oslo, Norway), Olivier Benveniste (Pitié-Salpêtrière Hospital, UPMC, Assistance Publique - Hôpitaux de Paris, Paris, France), Timothy Radstake (Department of Rheumatology and Clinical Immunology, University Medical Center Utrecht, Utrecht, The Netherlands), Jan De Bleecker, Boel De Paepe (Department of Neurology, Neuromuscular Reference Centre, Ghent University Hospital, Ghent, Belgium), Britta Maurer (Department of Rheumatology and Center of Experimental Rheumatology, University Hospital Zurich, Zurich, Switzerland), Leonid Padyukov (Rheumatology Unit, Department of Medicine, Karolinska University Hospital, Karolinska Institutet, Stockholm, Sweden), Terrance P. O'Hanlon (Environmental Autoimmunity Group, Clinical Research Branch, National Institute of Environmental Health Science, National Institutes of Health, Bethesda, MD, USA), Lucy R. Wedderburn (Arthritis Research UK Centre for Adolescent Rheumatology, and Institute of Child Health, University College London, London, UK), Christopher Denton (Royal Free Hospital, London, UK), Herman Mann (Institute of Rheumatology, Prague), 
David Hilton-Jones (John Radcliffe Hospital, Oxford, UK), Patrick Kiely (St. George's Hospital, London, UK), Paul H. Plotz (National Institute of Arthritis and Musculoskeletal and Skin Diseases, National Institutes of Health, Bethesda, MD, USA), Mark Gourley (National Institute of Arthritis and Musculoskeletal and Skin Diseases, National Institutes of Health, Bethesda, MD, USA), Galina Marder (North Shore University Hospital, Great Neck, NY, USA). Neil J. McHugh and Zoe E. Betteridge (Department of Pharmacy and Pharmacology, University of Bath, Bath, UK) generated the autoantibody data used in this study.

\section{Funding}

This study was supported by Myositis UK, Medical Research Council (MRC) UK grant MR/N003322/1; Arthritis Research UK (18474), Association Francaise Contre Les Myopathies (AFM), the European Union Sixth Framework Programme (project AutoCure; LSH-018661), the European Science Foundation (ESF) within the framework of the Research Networking Programme European Myositis Network (EUMYONET), the Swedish Research Council and the regional agreement on medical training and clinical research (ALF) between Stockholm County Council and Karolinska Institutet, and the intramural research program of the National Institute of Environmental Health Sciences (NIEHS) of the National Institutes of Health $(\mathrm{NIH})$. The Czech cohort was supported by Project for Conceptual Development of Research Organization 00023728 from the Ministry of Health in the Czech Republic. JEP is supported by a University of Manchester alumni "Research Impact" doctoral studentship and president's doctoral scholar award. This report includes description of independent research supported by the National Institute for Health Research. The views expressed in this publication are those of the author(s) and not necessarily those of the NHS, the National Institute for Health Research or the Department of Health.

\section{Availability of data and materials}

The data that support the findings of this study are available from the Myositis Genetics Consortium (MYOGEN), but restrictions apply to the availability of these data, which were used under licence for the present study, and so are not publicly available. Data are, however, available from the authors upon reasonable request and with permission of MYOGEN.

\section{Authors' contributions}

JEP contributed to the design of the study, performed the analyses, interpreted the data and wrote the manuscript. SR provided the data, assisted with data analysis and critically revised the manuscript. AO advised on statistical analysis, assisted with the interpretation of data and critically revised the manuscript. HC and JAL designed the study, assisted with acquisition of data, supervised the study and critically revised the manuscript. All authors read and approved the manuscript.

\section{Ethics approval and consent to participate}

Anonymised data were provided by the Myositis Genetics Consortium, which gained approval from research ethics boards at each participating centre and obtained informed consent from all participants for studies using this data.

\section{Competing interests}

The authors declare that they have no competing interests.

\section{Publisher's Note}

Springer Nature remains neutral with regard to jurisdictional claims in published maps and institutional affiliations.

\footnotetext{
Author details

${ }^{1}$ Centre for Epidemiology, Division of Population Health, Health Services Research \& Primary Care, Faculty of Biology, Medicine and Health, Manchester Academic Health Science Centre, University of Manchester, 2.706 Stopford Building, Oxford Road, Manchester M13 9PT, UK. ${ }^{2}$ Centre for Musculoskeletal Research, Division of Musculoskeletal \& Dermatological Sciences, Faculty of Biology, Medicine and Health, Manchester Academic Health Science Centre, University of Manchester, Manchester, UK. ${ }^{3}$ Arthritis Research UK Centre for Epidemiology, Centre for Musculoskeletal Research, University of Manchester, Manchester Academic Health Science Centre, Manchester, UK. ${ }^{4}$ National Institute for Health Research Manchester Biomedical Research Centre, Central Manchester University Hospitals NHS Foundation Trust, Manchester Academic Health Science Centre, University of
}

Manchester, Manchester, UK. ${ }^{5}$ Department of Rheumatology, Salford Royal NHS Foundation Trust, Manchester Academic Health Science Centre, Salford, UK.

Received: 21 February 2018 Accepted: 8 May 2018

Published online: 08 June 2018

\section{References}

1. GJD H, van Venrooij WJ, Vencovsky J, Moutsopoulos HM, van Engelen BGM. The relative prevalence of dermatomyositis and polymyositis in Europe exhibits a latitudinal gradient. Ann Rheum Dis. 2000;59:141-2. https://doi. org/10.1136/ard.59.2.141

2. Okada S, Weatherhead E, Targoff IN, Wesley R, Miller FW. Global surface ultraviolet radiation intensity may modulate the clinical and immunologic expression of autoimmune muscle disease. Arthritis Rheum. 2003:48:228593. https://doi.org/10.1002/art.11090.

3. Love LA, Weinberg CR, McConnaughey DR, Oddis V, Medsger TA, Reveille $J \mathrm{D}$, et al. Ultraviolet radiation intensity predicts the relative distribution of dermatomyositis and anti-Mi-2 autoantibodies in women. Arthritis Rheum. 2009;60:2499-504. https://doi.org/10.1002/art.24702.

4. Brouwer R, Hengstman GJD, Egberts WV, Ehrfeld H, Bozic B, Ghirardello A, et al. Autoantibody profiles in the sera of European patients with myositis. Ann Rheum Dis. 2001;60:116-23.

5. Cheong WK, Hughes RV, Norris PG, Hawk JLM. Cutaneous photosensitivity in dermatomyositis. Br J Dermatol. 1994;131:205-9.

6. Dourmishev L, Meffert H, Piazena H. Dermatomyositis: comparative studies of cutaneous photosensitivity in lupus erythematosus and normal subjects. Photodermatol Photoimmunol Photomed. 2004;20:230-4.

7. Matos TR, Sheth V. The symbiosis of phototherapy and photoimmunology. Clin Dermatol. 2016;34:538-47. https://doi.org/10.1016/..clindermatol.2016.05.003.

8. Rothwell S, Cooper RG, Lundberg IE, Miller FW, Gregersen PK, Bowes J, et al. Dense genotyping of immune-related loci in idiopathic inflammatory myopathies confirms HLA alleles as the strongest genetic risk factor and suggests different genetic background for major clinical subgroups. Ann Rheum Dis. 2016;75:155866. https://doi.org/10.1136/annrheumdis-2015-208119.

9. Gregersen PK, Lee HS, Batliwalla F, Begovich AB. PTPN22: setting thresholds for autoimmunity. Semin Immunol. 2006;18:214-23. https://doi.org/10.1016/ j.smim.2006.03.009

10. Mathieu A, Cauli A, Teresa M, Sorrentino R. HLA-B27 and ankylosing spondylitis geographic distribution as the result of a genetic selection induced by malaria endemic? A review supporting the hypothesis. Autoimmun Rev. 2008;7:398403. https://doi.org/10.1016/j.autrev.2008.03.013.

11. Rothwell S, Cooper RG, Lundberg I, et al. Focused HLA analysis in idiopathic inflammatory myopathy identifies significant associations of classical HLA alleles with autoantibody and clinical subgroups [abstract 770W]. Baltimore: Presented at the 65th annual meeting of the American Society of Human Genetics; 2015.

12. StataCorp. Stata Statistical Software: release 13. College Station: StataCorp LP; 2013.

13. Burd CJ, Kinyamu HK, Miller FW, Archer TK. UV radiation regulates Mi-2 through protein translation. J Biol Chem. 2008;283:34976-82. https://doi.org/ 10.1074/jbc.M805383200.

\section{Ready to submit your research? Choose BMC and benefit from:}

- fast, convenient online submission

- thorough peer review by experienced researchers in your field

- rapid publication on acceptance

- support for research data, including large and complex data types

- gold Open Access which fosters wider collaboration and increased citations

- maximum visibility for your research: over $100 \mathrm{M}$ website views per year

At BMC, research is always in progress.

Learn more biomedcentral.com/submissions 